\title{
TeV Neutrinos and GeV Photons from Shock Breakout in Supernovae
}

\section{Citation}

Waxman, Eli, and Abraham Loeb. 2001. "TeV Neutrinos and GeV Photons from Shock Breakout in Supernovae." Physical Review Letters 87 (7). https://doi.org/10.1103/physrevlett.87.071101.

\section{Permanent link}

http://nrs.harvard.edu/urn-3:HUL.InstRepos:41417279

\section{Terms of Use}

This article was downloaded from Harvard University's DASH repository, and is made available under the terms and conditions applicable to Other Posted Material, as set forth at http:// nrs.harvard.edu/urn-3:HUL.InstRepos:dash.current.terms-of-use\#LAA

\section{Share Your Story}

The Harvard community has made this article openly available.

Please share how this access benefits you. Submit a story.

\section{Accessibility}




\title{
TeV Neutrinos and GeV Photons from Shock Breakout in Supernovae
}

\author{
Eli Waxman ${ }^{\dagger} \&$ Abraham Loeb* \\ $\dagger$ Department of Condensed Matter Physics, Weizmann Institute, Rehovot 76100, Israel; waxman@wicc.weizmann.ac.il

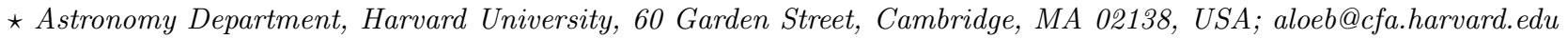

(December 2, 2018)

\begin{abstract}
We show that as a Type II supernova shock breaks out of its progenitor star, it becomes collisionless and may accelerate protons to energies $>10 \mathrm{TeV}$. Inelastic nuclear collisions of these protons produce $\mathrm{a} \sim 1 \mathrm{hr}$ long flash of $\mathrm{TeV}$ neutrinos and $10 \mathrm{GeV}$ photons, about $10 \mathrm{hr}$ after the thermal $(10 \mathrm{MeV})$ neutrino burst from the cooling neutron star. A Galactic supernova in a red supergiant star would produce a photon and neutrino flux of $\sim 10^{-4} \mathrm{erg}_{\mathrm{cm}}^{-2} \mathrm{~s}^{-1}$. A km $\mathrm{km}^{2}$ neutrino detector will detect $\sim 100$ muons, thus allowing to constrain both supernova models and neutrino properties.
\end{abstract}

PACS numbers: 97.60.Bw, 14.60.Pq, 98.70.Rz, 95.85.Ry

Type II supernovae are triggered by core collapse in a massive star, generating a strong shock wave which propagates through the progenitor star and ejects its envelope (see [1] for a review). Interest in the breakout of the shock out of the envelope of the star, was prompted by Colgate's suggestion that it may be accompanied by energetic $\gamma$-ray radiation due to Bremsstrahlung and inverseCompton emission of thermal electrons [2]. Numerical simulations concluded that the shock breakout should be accompanied by a burst of hard UV and X-ray radiation [3.

In the optically-thick interior of the progenitor, the shock is dominated by radiation. The dissipation of kinetic energy at the shock front is mediated by Compton scattering rather than by collisional viscosity, and the thermal energy density behind the shock is mostly in the form of radiation. Numerical simulations suggest that during breakout, the radiation-dominated shock is transformed into a viscous shock [3.4]. When the optical depth of the gas lying ahead of the shock is no longer very large, radiation decouples from the gas and the shock is transformed into a collisional, viscosity-mediated shock.

In this Letter, we show that as the shock becomes viscous, electro-magnetic instabilities at the shock front grow at a rate which is many orders of magnitude higher than the collision rate, leading to scattering of ions and to magnetic field amplification on a time scale much shorter than the viscous time scale. Under similar conditions, astrophysical shocks are known to become collisionless (i.e., to be mediated by collective plasma instabilities rather than by collisional viscosity) and to accelerate charged particles to relativistic energies (see [5] for a review). We derive the expected flux and spectrum of high energy photons and neutrinos which are produced through inelastic nuclear collisions of the accelerated protons.

As we show below, the resolution of available numerical simulations is not sufficient to correctly describe the evolution of plasma parameters across the viscous shock (see also [3]). We therefore first derive the expected collisional shock structure from basic principles, and only then discuss the stability of the shock. While our analysis provides a description of the shock structure, it does not allow to determine the exact radius where the viscous shock forms. Numerical calculations suggest that the transition to a viscosity-dominated shock occurs at an optical depth (measured from the stellar surface) between $\tau \sim 1$ [3] and $\tau \sim 10$ [4]. We therefore parameterize our results in terms of $\tau$. More detailed numerical simulations are necessary to allow an accurate determination of this parameter.

We focus our discussion on stars which are believed to be the most common progenitors of Type II supernovae, namely red-supergiants (RSG) [1]. Our model predicts emission of high energy particles also for blue-supergiants (BSGs), such as the unusual progenitor of SN1987A. However, the predicted neutrino and $\gamma$-ray fluxes from the surface layer of BSGs are predicted to be two orders of magnitude smaller than from RSGs. Additional emission may result from the interaction of the supernova shock with the progenitor wind on long time scales [6], although this emission depends sensitively on the wind speed and mass loss rate and might also be strongly modulated by past variability of the wind [7].

Physical parameters at shock breakout. When the radiation-dominated shock reaches an optical depth lower than unity, radiation can no longer couple to the gas and so the shock becomes viscous. This occurs very close to the surface radius of the progenitor star, $R_{\star}$. For the high temperatures of interest, the opacity is dominated by Thomson scattering, and the mass of a shell of optical depth $\tau$ located ahead of the shock is given by

$$
M=4 \pi R_{*}^{2} \frac{\mu m_{p}}{\sigma_{T}} \tau=1.8 \times 10^{-5} R_{*, 13.5}^{2} \tau M_{\odot},
$$

where $R_{\star, 13.5} \equiv\left(R_{\star} / 10^{13.5} \mathrm{~cm}\right)=1$ reflects the characteristic radius of a RSG progenitor. As mentioned above, we ignore rarer, more compact progenitors, such as blue supergiants for which $R_{\star, 13.5}=0.1$, since the shell mass at a fixed $\tau$, and hence the energy output in high-energy neutrinos and photons, are smaller by a factor $\sim 10^{2}$ in them compared to RSGs. Using the analytic approxi- 
mations of Matzner \& Mckee [8] to describe the outer envelope structure, we find that the mass density of gas at the RSG surface is

$$
\rho=0.54 \times 10^{-10} \tau^{3 / 5} \mathrm{~g} \mathrm{~cm}^{-3},
$$

and the shell thickness is $\Delta=1.1 \times 10^{11} \tau^{2 / 5} \mathrm{~cm}$.

The shock velocity at breakout is $v_{s} \approx 10^{9} \mathrm{~cm} \mathrm{~s}^{-1}$ (e.g. [8]), corresponding to an ion temperature of

$$
T_{i}=\frac{3}{16} \mu v_{s}^{2}=1.0 \times 10^{5} v_{s, 9}^{2} \mathrm{eV},
$$

where $\mu=m_{p} / 2$ is the mean atomic mass per particle for a plasma in which the thermal energy is shared equally between protons and electrons. If the shock is radiationdominated, the radiation temperature is approximately given by the relation $a T_{r}^{4} \approx \rho v_{s}^{2}$, i.e.

$$
T_{r} \approx 30 \rho_{-10}^{1 / 4} v_{s, 9}^{1 / 2} \mathrm{eV},
$$

where $\rho_{-10}=\left(\rho / 10^{-10} \mathrm{~g} \mathrm{~cm}^{-3}\right)$.

Viscous shock structure. The ion-ion, ion-electron, and electron-electron collision rates are given by

$$
\begin{gathered}
\nu_{i i} \approx 3 \rho_{-10} T_{i, 5}^{-3 / 2} \mathrm{~s}^{-1}, \\
\nu_{i e} \approx 10^{3} \rho_{-10} \times \begin{cases}6 T_{i, 5}^{-3 / 2} \mathrm{~s}^{-1}, & \text { if } T_{e}<m_{e} T_{i} / m_{p} ; \\
2 T_{e, 2}^{-3 / 2} \mathrm{~s}^{-1}, & \text { otherwise },\end{cases} \\
\nu_{e e} \approx 10^{4} \rho_{-10} T_{e, 4}^{-3 / 2} \mathrm{~s}^{-1} .
\end{gathered}
$$

Throughout this Letter, we use the notation $T_{\alpha, x}=$ $\left(T_{\alpha} / 10^{x} \mathrm{eV}\right)$ to denote the temperature of component $\alpha$ (=i for ions, $e$ for electrons, or $r$ for radiation) of the plasma. The collisional energy loss of ions is dominated by ion-ion collisions once the electrons are heated to a temperature $T_{e} \gtrsim 0.07 T_{i}$.

The electrons cool by Compton scattering and Bremsstrahlung emission. Compton scattering dominates for $T_{e} \gtrsim 10^{2} \mathrm{eV}$, and provides a cooling rate of $\nu_{\text {Comp }}=\left(8 \sigma_{T} / 3 m_{e} c\right) a T_{r}^{4}=8 T_{r, 1.5}^{4} \mathrm{~s}^{-1}$. The electron temperature is determined by the balance between heating and cooling, $\nu_{i e} T_{i} \approx \nu_{\mathrm{Comp}} T_{e}$, yielding a post-shock electron temperature

$$
T_{e+} \approx 3 \times 10^{4}\left(T_{i, 5} \rho_{-10}\right)^{2 / 5} T_{r, 1.5}^{-8 / 5} \mathrm{eV} .
$$

At this temperature, the ion-electron collision rate is

$$
\nu_{i e+} \approx 0.4 T_{i, 5}^{-3 / 5} \rho_{-10}^{2 / 5} T_{r, 1.5}^{12 / 5} \mathrm{~s}^{-1} .
$$

Based on Eqs. (5)-(9), we conclude that the ion kinetic energy dissipation is dominated by ion-ion collisions, leading to a shock front width $\Delta_{s, i i} \approx \nu_{i i}^{-1} v_{s}=$ $3 \times 10^{8} \rho_{-10}^{-1} \mathrm{~cm}$. The electrons are heated by the ions to a temperature $T_{e+} \approx 30 \mathrm{keV}$ which is lower by a factor of a few than the ion temperature, $T_{i} \approx 100 \mathrm{keV}$. Behind the shock, the electrons cool by Compton scattering, but their temperature is maintained at $T_{e} \approx T_{e+}$ over a shell of width $\approx \nu_{i e}^{-1} v_{s} \approx 10^{9} \rho_{-10}^{-2 / 5} \mathrm{~cm}$, due to their coupling to the ions. Over a larger length scale, both the electrons and ions cool below their post shock temperature. The electrons maintain a local thermodynamic equilibrium, since they are heated to $T_{e} \gtrsim 0.07 T_{i}$ over a length scale $\ll \Delta_{s, i i}$ and subsequently $\nu_{e e} \gg \nu_{i e}$.

Our results agree with the numerical simulations of Klein \& Chevalier [3] for a RSG progenitor, who concluded that a collisional shock is formed at $\tau \sim 1$ where $T_{r} \sim 10 \mathrm{eV}$. Klein \& Chevalier derived a post-shock temperature of $\sim 10^{4} \mathrm{eV}$, but pointed out that this result depends on the artificial viscosity they used, with higher temperatures reached for lower artificial viscosity values (which yield a sharper shock), and thus concluded that the gas should be heated up in reality to $T \approx 10^{5} \mathrm{eV}$. Ensman \& Burrows [4] found in their simulations of a BSG progenitor that a collisional shock forms at $\tau>10$ but reaches lower temperatures, $\sim 1 \mathrm{keV}$. This calculation, however, assumed thermal equilibrium between the ions and the electrons. Unfortunately, the resolution of all numerical simulations so far has not been sufficient to correctly determine the balance between collisional heating and radiative cooling on the relevant length scale of $\sim 10^{8} \mathrm{~cm}$.

Electro-magnetic stability and the collisionless shock. The ion velocity distribution within the collisional shock front is highly anisotropic over a length scale $\Delta_{s, i i}$. This anisotropy leads to the development of electro-magnetic instabilities. In order to analyze the shock front stability, we consider a plasma composed of electrons in thermal equilibrium at a temperature $T_{e}$, and protons with a bi-Maxwellian distribution having a temperature $T_{\perp}=m_{p} v_{s}^{2}$ along one axis (representing the velocity dispersion associated with the ion streams perpendicular to the shock front), and a lower temperature in the orthogonal plane $T_{i}<T_{\perp}$ (parallel to the shock front). Since the electrons in our case have large thermal speeds, namely $T_{e} / T_{\perp} \sim 0.1 \gg m_{e} / m_{p}$, electrostatic instabilities such as the two-stream instability, are suppressed. We therefore focus on electro-magnetic instabilities driven by the ion anisotropy. Such instabilities are typically expected to grow at a rate of $\leq\left(v_{s} / c\right) \nu_{p i}$, where $\nu_{p i}$ is the ion plasma frequency (e.g. [9]), with the maximum rate obtained only in the limit of infinite $T_{e}$.

Solving the dispersion relation for electro-magnetic waves in the above plasma, we find purely growing modes (i.e. modes having purely imaginary frequencies) with an electric field along the direction of the high ion temperature (i.e. perpendicular to the shock front), as long as the wave vector $k$ satisfies the condition $\left(k c / 2 \pi \nu_{p i}\right)^{2}<$ $\left(T_{\perp} / T_{i}\right)-1$. In the limit $m_{e} / m_{p} \ll T_{e} / T_{\perp} \ll m_{p} / m_{e}$, 
the growth rates are

$\nu_{E M} \approx \frac{v_{s}}{c} \nu_{p i} \times \begin{cases}\min \left(1, k / Y^{1 / 2} k_{*}\right), & \text { for } T_{\perp} / T_{i} \gg Y ; \\ \left(\frac{T_{\perp}}{T_{i}}-1\right) k / Y^{3 / 2} k_{*}, & \text { for } T_{\perp} / T_{i} \ll Y,\end{cases}$

where $k_{*}=2 \pi \nu_{p i} / c$ and $Y=\left(m_{p} T_{\perp} / m_{e} T_{e}\right)^{1 / 3}$.

For large anisotropy, $T_{\perp} / T_{i} \gg Y$, the maximal growth rate is $\left(v_{s} / c\right) \nu_{p i}$. If the anisotropy is smaller, the maximal growth rate is $\left(v_{s} / c\right) \nu_{p i} / Y^{3 / 2}$, which in our case yields

$$
\nu_{E M} \approx\left(0.1 \frac{m_{e}}{m_{p}}\right)^{1 / 2} \frac{v_{s}}{c} \nu_{p i} \approx 3 \times 10^{6} \rho_{-10}^{1 / 2} v_{s, 9} \mathrm{~s}^{-1} .
$$

The growth rate of electro-magnetic instabilities is therefore many orders of magnitude larger than the collision rates $\nu_{i i}, \nu_{i e}$. Correspondingly, the characteristic length scale of the fastest growing modes, $c \nu_{p i}^{-1}=20 \rho_{-10}^{1 / 2} \mathrm{~cm}$, is many orders of magnitude smaller than the collisional shock width. Under such conditions, we expect the shock to become collisionless, i.e. to be mediated by plasma instabilities rather than by collisions. The Ohmic dissipation rate of the electro-magnetic fields, $\lesssim$ $\left(m_{e} / m_{p}\right)\left(\ell / c \nu_{p i}^{-1}\right)^{-2} \nu_{e e}$, is much lower than their growth rate for all relevant temperatures and length scales $\ell \gtrsim$ $c \nu_{p i}^{-1}$.

If a collisionless shock indeed forms, it would accelerate charged particles to high energies, producing a power-low distribution of particle number, $d N / d p \propto p^{-2}$ where $p$ is the particle momentum [5]. The acceleration rate of either relativistic or non-relativistic protons is given by, $\nu_{\text {acc }} \sim \nu_{B}\left(E / E_{\mathrm{th}}\right)^{-1}$, where $E$ is the kinetic energy of the accelerated proton, $E_{\mathrm{th}}$ is the energy of thermal protons, and $\nu_{B}$ is the (non-relativistic) proton gyro-frequency,

$$
\nu_{B}=\frac{1}{2 \pi} \frac{e B}{m_{p} c}=1.5 \times 10^{4} B_{1} \mathrm{~s}^{-1},
$$

where $B_{1}=(B / 10 \mathrm{G})$ is the magnetic field normalized to $10 \mathrm{G}$, which is the typical value inferred at the surface of RSGs [10]. Note, however, that the electromagnetic instabilities build-up the magnetic field. In analogy with collisionless shocks in supernova remnants [11], $\gamma$-ray bursts [12], or the intergalactic medium [13], the magnetic field may grow up to a sizeable fraction of the equipartition value, $B_{\text {e.p. }}=10^{4.5} \rho_{-10}^{1 / 2} v_{s, 9} \mathrm{G}$, and saturate near this value due to nonlinear effects [14]. Thus, the acceleration rate is likely to significantly exceed the rate implied by Eq. (12). Since the acceleration rate at thermal energies, $\nu_{B}$, is much higher than the collision frequency of thermal protons, and since the collisional energy loss rate decreases as $E^{-3 / 2}$ while the acceleration rate declines only as $E^{-1}$, protons are expected to be accelerated to energies well above thermal.

Scattering of protons by magnetic field fluctuations is most effective if the fluctuation wavelength is compara- ble or larger than the proton Larmor radius. The Larmor radius of the thermal protons in an equipartition field is comparable to the wavelength $c \nu_{p i}^{-1}$ over which instabilities grow fastest. As the Larmor radius of highly relativistic protons is much larger, a conservative lower limit to the maximum energy of the accelerated protons is given by

$$
E_{\max } \approx \frac{v_{s}}{c} e B \Delta=18 v_{s, 9} B_{1} \tau^{2 / 5} \mathrm{TeV},
$$

where we have assumed that instabilities do not lead to significant amplification of the field on large scales. This is probably an underestimate of the maximum energy, as nonlinear effects are expected to amplify the field on scales $\gg c \nu_{p i}^{-1}$. If this occurs, then protons would be accelerated up to energies well in excess of $10 \mathrm{TeV}$.

Neutrinos and photons. Protons accelerated to relativistic energy lose energy by inelastic nuclear collisions. The average number of nuclear collisions encountered by a proton is $N_{c} \approx n \sigma_{0} c \Delta / v_{s}=5.2 \tau / v_{s, 9}$, where $\sigma_{0}=50 \mathrm{mb}$. Since a proton loses $\sim 20 \%$ of its energy in each collision, the total energy lost to pion production is

$$
E_{\pi} \approx \xi_{p} \min (1, \tau) \frac{M}{m_{p}} T_{i}=3 \xi_{p} \min \left(\tau, \tau^{2}\right) \times 10^{45} \mathrm{erg}
$$

where $\xi_{p}$ is the fraction of the post-shock energy density which is converted to relativistic protons (note that for a spectrum $d N / d p \propto p^{-2}$, the energy of super-thermal particles is dominated by relativistic particles). For the strong shocks around supernova remnants, the inferred values of $\xi_{p}$ are of order unity [15].

Roughly a third of $E_{\pi}$ is converted to muon neutrinos, and a similar fraction to high energy photons, through pion decay. The neutrino and photon signals should be coincident and spread over a time scale of $2 R_{*} / c \approx 1$ hour. (The emission from the back side of the progenitor star is visible for $\mathrm{TeV}$ neutrinos but not so for $\gamma$-rays, and consequently the $\gamma$-ray flash should be shorter by about a factor of two than the neutrino pulse.) The predicted flux of $\gamma$-rays and muon neutrinos from a Type II supernova in our Galaxy is therefore

$$
F_{\gamma} \approx F_{\nu_{\mu}} \approx 10^{-4} \xi_{p} \min \left(\tau, \tau^{2}\right) d_{10 \mathrm{kpc}}^{-2} \operatorname{erg~s}^{-1} \mathrm{~cm}^{-2},
$$

where $d_{10 \mathrm{kpc}}$ is the supernova distance in units of $10 \mathrm{kpc}$. The high density of $\sim 10 \mathrm{eV}$ photons from the radiative shock, will lead to a high pair-production optical depth for photons with energies $\gtrsim 10 \mathrm{GeV}$. Thus, we expect a photon spectrum $d N_{\gamma} / d E_{\gamma} \propto E_{\gamma}^{-2}$ over the energy range $100 \mathrm{MeV} \lesssim E_{\gamma} \lesssim 10 \mathrm{GeV}$. This signal is easily detectable with planned $\gamma$-ray telescopes (e.g. [16]).

The neutrino spectrum is expected to follow the accelerated proton spectrum, with a number per energy 
interval of $d N / d E \propto E^{-2}$ at relativistic proton energies. The probability that a muon neutrino will produce a high-energy muon in a terrestrial detector is 17 $P_{\nu \mu} \approx 1.3 \times 10^{-6} E_{\nu, \mathrm{TeV}}^{\beta}$, with $\beta=2$ for $E_{\nu, \mathrm{TeV}}<1$ and $\beta=1$ for $E_{\nu, \mathrm{TeV}}>1$. Thus, the number flux $J_{\mu}$ of muon induced neutrinos can be related to the neutrino energy flux, $F_{\nu}=\int d E_{\nu} E_{\nu}\left(d J_{\nu} / d E_{\nu}\right)$, where $J_{\nu}$ is the neutrino number flux, through the relation

$$
J_{\mu}=\int d E_{\nu} \frac{d J_{\nu}}{d E_{\nu}} P_{\nu \mu} \approx \frac{1+\ln \left(E_{\nu, \max } / 1 \mathrm{TeV}\right)}{\ln \left(E_{\nu, \max } / 1 \mathrm{GeV}\right)} \frac{P_{0}}{E_{0}} F_{\nu},
$$

where $P_{0} / E_{0}=1.3 \times 10^{-6} \mathrm{TeV}^{-1}$. Using Eq. (14) we find that

$$
N_{\mu} \approx 0.15 \frac{P_{0}}{E_{0}} \frac{E_{\pi} / 3}{4 \pi d^{2}}=130 \xi_{p} \min \left(\tau, \tau^{2}\right) d_{10 \mathrm{kpc}}^{-2} \mathrm{~km}^{-2}
$$

muons are expected to be detected for a single supernova. The factor 0.15 follows from Eq. (16) under the assumption $E_{\nu, \max } \approx 1 \mathrm{TeV}$. This is probably a conservative estimate, as proton energies $E_{p} \gg 10 \mathrm{TeV}$ may be achieved if the magnetic field is amplified.

Implications. A type II supernova in our Galaxy, which should occur once per 40 years [18], is expected to produce a strong neutrino signal of $\sim 100$ events at $\sim 1 \mathrm{TeV}$ spread over $\sim 1 \mathrm{hr}$, in planned square-kilometer neutrino detectors (see, e.g., [19] for a review). This is the strongest astrophysical signal predicted for the next generation of $\mathrm{TeV}$ neutrino detectors. Detection of the neutrino signal would be feasible also for optically-dark supernovae in the Galactic center or in molecular clouds, which are heavily obscured by dust. Combined analysis of the detected signal along with the thermal (10 $\mathrm{MeV}$, few seconds long) neutrino burst from the cooling neutron star which should occur about 10 hours earlier $\left(\sim R_{\star} / v_{s}\right)$, could provide important clues about the formation process of the neutron star and the dynamics of the resulting supernova shock.

For the neutrino parameters inferred from recent atmospheric experiments 20], we expect flavor oscillations to produce equal fluxes of muon and tau neutrinos upon their arrival to Earth, thus allowing for a " $\tau$ appearance" experiment. Since the neutrino signal should coincide with the $\gamma$-ray signal, checking the simultaneity of photon and neutrino arrival times will allow to test for deviations from Lorentz invariance and from the weak equivalence principle. Although the neutrino and photon signals are expected to spread over $\sim 1 \mathrm{hr}$, detection of a large number of neutrinos will allow to test simultaneity to an accuracy much better then $1 \mathrm{hr}$, thus providing better limits than those derived from supernova 1987A, where simultaneity was tested to an accuracy of only several hours (see [21] for a review).

Acknowledgments. We thank Z. Barkat, S. Blinnikov, A. Burrows, R. Chevalier, M. Reid and S. Saar for useful discussions. This work was supported in part by grants from the Israel-US BSF (BSF-9800343), MINERVA, NSF (AST-9900877; AST-0071019), and NASA (NAG5-7039; NAG5-7768). AL thanks the Weizmann Institute for its kind hospitality during the course of this work.

[1] S. E. Woosley \& T. A. Weaver, Ann. Rev. Astron. Astrophys. 24, 205 (1986)

[2] S. A. Colgate, Ap.J. 187, 333 (1974).

[3] R. I. Klein, \& R. A. Chevalier, Astrophys. J. 223, L109 (1978); 234, 597 (1979).

[4] L. Ensman, \& A. Burrows, Astrophys. J. 393, 742 (1992).

[5] R. Blandford, \& D., Eichler, Phys. Rep. 154, 1 (1987).

[6] E. G. Berezhko, \& H. J. Völk, Astr. \& Astrophys., 357, 283 (2000), and references therein. See also V. S. Berezinskii, \& V. L. Ginzburg, Nature, 329, 807, (1987).

[7] T. Bloecker, Y. Balega, K.-H. Hofmann, \& G. Weigelt Astr. \& Astrophys., in press, 2001 (astro-ph/0102092),

[8] C. D. Matzner, \& C. F. McKee, Astrophys. J. 510, 379 (1999).

[9] N. Krall \& Trivelpiece Principles of plasma physics (New York: McGraw-Hill, 1973).

[10] M. J. Reid, in IAU Symp. No. 140, Galactic and Extragalactic Magnetic Fields, Ed. R. Beck, P. P. Kronberg, \& R. Wielebinski, (Kluwer Academic Pub; Dordrecht 1990), p. 21-25; M. Reid, et al., Astroph. J., 227, L89 (1979); R. Barvainis, G. McIntoch, \& C. R. Predmore, Nature, 329, 613 (1987); A. J. Kemball, \& P. J. Diamond, Astroph. J., 481, L111 (1997).

[11] D. J. Helfand and R. H. Becker, Astrophys. J., 314, 203 (1987); P. J. Cargill and K. Papadopoulos, Astrophys. J., 329, L29 (1988).

[12] M. V. Medvedev, \& A. Loeb, Astrophys. J., 526, 697 (1999); A. Gruzinov \& E. Waxman, Astrophys. J., 511, 852 (1999); D. Medvigy, \& A. Loeb, submitted to Phys. Rev. E, 2000 (astro-ph/0012029.

[13] A. Loeb, \& E. Waxman, Nature, 405, 156 (2000); E. Waxman, \& A. Loeb, Astrophys. J. Lett., 545, L11 (2000).

[14] F. Califano et al., Phys. Rev. E 57, 7048 (1998); Y. Kazimura et al., Ap. J., 498, L183 (1998); T.-Y. Yang, J. Arons, \& A. B. Langdom, Phys. Plasmas, 1, 3059 (1994); J. M. Wallace, \& E. M. Epperlein, Phys. Fluids, 3, 1579 (1991).

[15] For shock-accelerated electrons, relativistic energy fractions $\xi_{e}$ of order a few percent are inferred from X-ray and $\mathrm{TeV}$ observations [e.g. A. Mastichiadis \& O. C. de Jager, Astron. Astrophys. 311, L5 (1996); T. Tanimori et al., Astrophys. J. 497, L25 (1998)], and for relativistic protons, $\xi_{p} \gtrsim 10 \xi_{e}$ based on cosmic-ray observations (e.g. (5).

[16] See, e.g. http://glast.gsfc.nasa.gov/ (GLAST), and http://www.ifctr.mi.cnr.it/Agile/ (AGILE).

[17] T. K. Gaisser, F. Halzen, and T. Stanev, Phys. Rep. 258, 173 (1995). 
[18] G. A. Tammann, in Supernovae: A survey of current research, eds. M. J. Rees \& R. J. Stoneham (Dordrecht: Reidel 1982).

[19] F. Halzen, in 17th Intl. Workshop on Weak Interactions and neutrinos, Cape Town, South Africa 1999 astro$\mathrm{ph} / 9904216$ ).

[20] Y. Fukuda et al., Phys. Lett. B335, 237 (1994); D. Casper et al., Phys. Rev. Lett. 66, 2561 (1991); G. L. Fogli, and E. Lisi, Phys. Rev. D52, 2775 (1995).

[21] J. N. Bahcall, Neutrino Astrophysics, Cambridge University Press (NY 1989), pp. 438-460. 